\title{
Evaluating the launch of live electronic access to point-of- care blood glucose monitoring to reduce the prevalence of inpatient hypoglycaemia at Heartlands, Good Hope and Solihull hospitals
}

\author{
Authors: Adeeba Ahmed, ${ }^{\mathrm{A}}$ Elizabeth Morley, ${ }^{\mathrm{B}}$ Farah Abdel Hameed, ${ }^{\mathrm{B}}$ Gayle Reynolds, ${ }^{\mathrm{A}}$ Michele Colloby, ${ }^{\mathrm{A}}$ \\ Catherine Holmes, ${ }^{A}$ Andrew Woodburn Drayton, ${ }^{A}$ Natasha Jacques, ${ }^{A}$ Ali Bahron ${ }^{A}$ and Sudarshan Ramachandran ${ }^{A}$
}

\section{Introduction}

Hypoglycaemia is a serious complication of diabetes, increasing risk of morbidity, mortality and length of stay. Live point-of-care (POCT) blood glucose monitoring provides a graphical-visual comparison of glucose control. Successive national diabetes inpatient audits have shown a significant level of mild and moderate hypoglycaemia for inpatients with diabetes in our hospitals, on par with other hospital trusts nationally. Many of these hospital trusts already have live point-of-care blood glucose connectivity, and we were keen to ensure that our switch was linked to a sustained reduction in patient harm from hypoglycaemia. Therefore, we planned to link the launch to a full communication plan to all inpatient areas during insulin safety week in 2018, along with a QI plan to intelligently target diabetes inpatient team reviews to patients with recurrent or severe hypoglycaemia.

\section{Methods}

A high-level collaborative project between the diabetes team, POCT team and IT allowed the electronic system (transferring the patient's POCT blood glucose results to their electronic record) to go live in March 2018. The system also reports recurrent and severe hypoglycaemic readings to a separate worklist on our diabetes electronic referral system, allowing these patients to be targeted for review by the inpatient diabetes team, even before referral has been received. We also communicated the new system to all clinical staff during insulin safety week in May 2018, with quick reference cards, screensavers, and a ward insulin safety board competition with a good participation rate from inpatient wards.

We retrospectively audited the electronic records from 01-28 February 2018 (before the launch of the new system) and from 01-28 October 2018 (7 months after the launch of the system) across the three hospital sites (an analysis of over 800 patient records), looking at the prevalence of mild $(3.0-3.9 \mathrm{mmol} / \mathrm{L})$ and severe $(<3.0 \mathrm{mmol} / \mathrm{L})$ hypoglycaemic episodes among inpatients

Authors: AUniversity Hospitals Birmingham NHS Foundation Trust, Birmingham, UK; ${ }^{B}$ University of Birmingham, Birmingham, UK



Fig 1. Timed ( $x$ axis) episodic prevalence ( $y$ axis) of severe hypoglycaemia ( $<3 \mathrm{mmol} / \mathrm{L}$ ) over 4 weeks in February 2018 and October 2018.

with diabetes on all three hospital sites. Patients without diabetes or not on hypoglycaemic medications were excluded.

\section{Results and discussion}

Between February and October 2018, the prevalence of total hypoglycaemic events decreased by $50 \%$ from 848 to 423 events,

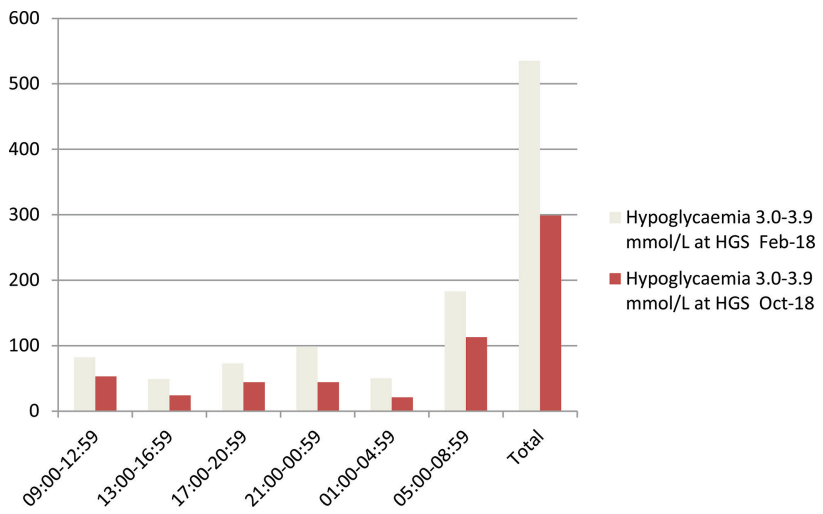

Fig 2. Timed ( $x$ axis) episodic prevalence (y axis) of moderate hypoglycaemia (3.0-3.9 mmol/L) over 4 weeks in February 2018 and October 2018. 
mild hypoglycaemic events decreased by $44 \%$ from 535 to 299 events, and severe hypoglycaemic events decreased by $60 \%$ from 313 to 124 events (Figs 1 and 2). The highest prevalence of mild and severe events occurred during the early morning period 05:00-08:59 in February and October $(p<0.05)$.

\section{Conclusion}

Our results showed a sustained and statistically significant reduction in patient harm from hypoglycaemia with the new pointof-care glucose connectivity and QI work to intelligently target at-risk patients. The system offers an efficient and reliable method for early escalation before a referral may have been made by the parent team, and encourages ward staff to take better ownership of their patients' diabetes management. We have since extended the project further with a pilot project for prescription bedtime snacks for at-risk patients.

\section{Conflicts of interest}

None declared. 